\title{
Efecto de Niveles Creciente de NPK más Microelementos en la Producción de Pasiflora Edulis, en Colonche, Santa Elena
}

\author{
Ing. Ángel León Mejía. ${ }^{(1)}$, Romer Suárez C. ${ }^{(2)}$, Gabriel Tomalá F. ${ }^{(3)}$ \\ Centro de Investigaciones Agropecuarias ${ }^{(1)}$ \\ Facultad de Ciencias Agrarias ${ }^{(2,3)}$ \\ Universidad Estatal Península (UPSE) de Santa Elena \\ Campus La Libertad, vía principal Santa Elena- La Libertad \\ La Libertad - Ecuador \\ arlem1969@yahoo.es
}

\begin{abstract}
Resumen
El ensayo se lo realizó en la comuna San Vicente de Colonche, teniendo como objetivo evaluar el efecto de niveles crecientes de nitrógeno, fósforo y potasio más microelementos en la producción de Passiflora edulis. Se probaron 16 niveles de $\mathrm{N}-\mathrm{P}_{2} \mathrm{O}_{5}-\mathrm{K}_{2} \mathrm{O}$ con tres repeticiones; diseño estadístico utilizado bloques completos al azar y las medias comparadas según rangos múltiples de Duncan al 5\%. Fertilización realizada cada dos meses después del trasplante; variables agronómicas evaluadas: altura de planta, diámetro del fruto, peso del fruto, rendimiento por hectárea y análisis económico. Los resultados mostraron que las dosis de fertilizantes influyen positivamente en el comportamiento productivo del cultivo, El tratamiento T9 (150N; $90 \mathrm{P}_{2} \mathrm{O}_{5} ; 100 \mathrm{~K}_{2} \mathrm{O}$ ) alcanzó el mejor rendimiento con $15574,47 \mathrm{~kg} / \mathrm{ha}$, pero los tratamientos 11,6 , y 10 superan la tasa de retorno mínima aceptable considerada en $100 \%$.
\end{abstract}

Palabras claves: Passiflora, fertilización, nutrientes, manejo, rendimiento.

\begin{abstract}
A field experiment was carried out, in San Vicente de Colonche. The objective of this study was to evaluate the effect of different doses of nitrogen, phosphorus and potassium more microelements alone or in combination in Passiflora edulis. There were used 16 doses of $\mathrm{N}-\mathrm{P}_{2} \mathrm{O} 5-\mathrm{K}_{2} \mathrm{O}$. It was used a randomized complete block design and the averages compared by Duncan's multiple range 5\%. The application frequency after transplant was every two months; evaluated agronomic variables: plant height, fruit diameter, fruit weight, yield per plant and economic analysis. The results demonstrate that management of doses of rate of fertilizers can influence the productive behavior of the Passiflora. The best treatment was T9 150N; $90 \mathrm{P}_{2} \mathrm{O} 5 ; 100 \mathrm{~K}_{2} \mathrm{O}$ ) with 15574, 47 kg/ha, however the treatments 11, 6 and 10 exceeded the acceptable minimum return rate marginal considered in $100 \%$.
\end{abstract}

Keywords: Passiflora, fertilization, nutrients, management, yield 


\section{Introducción}

La mayor superficie cultivada de maracuyá se encuentra localizada en la costa (Cuadro 1); el área sembrada se aproxima a las 26000 hectáreas con una producción promedio de 212000 toneladas métricas y rendimiento de $8 \mathrm{t} / \mathrm{ha}$. Las provincias donde se concentra el mayor hectareaje y producción de maracuyá son Los Ríos, Manabí, Guayas y Esmeraldas (1).

Cuadro 1. Provincias de mayor producción de maracuyá

\begin{tabular}{|c|c|c|c|}
\hline Provincias & $\begin{array}{c}\text { Superficie } \\
\text { (ha) }\end{array}$ & $\begin{array}{c}\text { Producción } \\
\text { (t) }\end{array}$ & $\begin{array}{c}\text { Rendimiento } \\
\text { (t/ha) }\end{array}$ \\
\hline Los Ríos & 18605 & 204013 & 11,00 \\
\hline Manabí & 4481 & 27407 & 6,12 \\
\hline Guayas & 2309 & 9200 & 3,98 \\
\hline Esmeraldas & 1514 & 5698 & 3,76 \\
\hline
\end{tabular}

Fuente: III Censo Nacional Agropecuario

En el período 1995-2004, la exportación de maracuyá generó 260 millones de dólares en divisas, siendo Holanda el más alto consumidor de jugo concentrado (2). En el 2008, 18 mil toneladas de concentrado (sin agua y azúcar) compraron embotelladoras de la industria de jugos en el mundo, para elaborar mezclas de jugos tropicales y bebidas. Las exportaciones a Holanda, Estados Unidos, Australia, Sudáfrica, Reino Unido, España generaron \$ 60476000 (3).

La demanda de fruta en el Ecuador, por las plantas procesadoras, supera la producción, pues en la actualidad, es el primer país exportador de jugo concentrado de maracuyá a nivel mundial.

Alrededor de 50000 familias viven del cultivo de maracuyá, de allí la importancia socio económica del cultivo, sobre todo, en fincas pequeñas en las que se involucra la familia en todo el proceso productivo (4).

En la provincia de Santa Elena, existen alrededor de 300 hectáreas de maracuyá ubicadas en varias zonas, en la que se aplica tecnología no validada, si se considera las particularidades de clima y suelo de la península (5). La fertilización dentro de un sistema de producción es uno de los aspectos más importantes para el rendimiento máximo de un cultivo, razón por el cual es necesario conocer el nivel natural de los nutrientes en el suelo, con el propósito de establecer programas de fertilización que eleven o por lo menos conserven la fertilidad (6).

Por lo tanto, al elaborar un sistema o plan de fertilización se deben conocer y relacionar todos los factores que intervienen en el proceso de nutrición vegetal, cultivo, suelo, fertilizantes, épocas y dosis de aplicación.

Las características agroclimáticas de la península de Santa Elena, hacen que este cultivo se torne en una buena alternativa para la diversificación de la producción agrícola, siendo necesario estudios que permitan elaborar un paquete técnico acorde con las características de la región.

La fertilización es uno de los parámetros de gran importancia en el manejo técnico del cultivo, por esta razón la UPSE a través del CIAP se propone realizar trabajos de investigación relacionados con sistemas de nutrición en el cultivo de maracuyá en las zonas productoras de la provincia.

\section{Materiales y métodos}

El trabajo de investigación se ejecutó en la Comuna las Balsas, parroquia Colonche, cantón Santa Elena; pluviometría media anual $486 \mathrm{~mm}$, temperatura media anual 24,12 ${ }^{\circ} \mathrm{C}$; según la clasificación climática de Holdridge corresponde a bosque espinoso.

Suelo arcilloso, pH 6,2; Nitrógeno 10 ppm; Fósforo 9 ppm; Potasio 0,63 meq; Conductividad eléctrica 0,26 ds/m; RAS 2,4; PSI 2,5.

\subsection{Material biológico}

Variedad INIAP 2009 desarrollado por Estación Experimental de INIAP Portoviejo, liberada en diciembre del 2009.

\subsection{Biometría}

2.3.1 Factores en estudio. Niveles de fertilización NPK más microelementos.

2.3.2 Tratamientos. El experimento se diseñó con 16 tratamientos, de los cuales cuatro se complementaron con aplicaciones foliares de boro, zinc y azufre. Fuentes de fertilizantes: Nitrofoska especial (12-12-17+ Mg $2 \%$, anhidro sulfúrico $20 \%$, B 0,02 \%, Zn 0,01 $\%)$; MAP (11-52); nitrato de amonio (N 33,5 \%), nitrato de potasio ( $13-50$ ), kelatox boro, kelatox zinc, cuadro 2.

Cuadro 2. Tratamientos

\begin{tabular}{|c|c|c|c|}
\hline Tratamientos & $\begin{array}{c}\mathrm{N} \\
\mathrm{kg} / \mathrm{ha}\end{array}$ & $\begin{array}{l}\mathrm{P}_{2} \mathrm{O}_{5} \\
\mathrm{~kg} / \mathrm{ha}\end{array}$ & $\begin{array}{l}\mathrm{K}_{2} \mathrm{O} \\
\mathrm{kg} / \mathrm{ha} .\end{array}$ \\
\hline $\mathrm{T} 1$ & 0 & 0 & 0 \\
\hline $\mathrm{T} 2$ & 100 & 50 & 100 \\
\hline $\mathrm{T} 3$ & 150 & 50 & 100 \\
\hline $\mathrm{T} 4$ & 200 & 50 & 100 \\
\hline $\mathrm{T} 5$ & 250 & 50 & 100 \\
\hline $\mathrm{T} 6$ & 150 & 40 & 100 \\
\hline $\mathrm{T} 7$ & 150 & 60 & 100 \\
\hline $\mathrm{T} 8$ & 150 & 80 & 100 \\
\hline $\mathrm{T} 9$ & 150 & 90 & 100 \\
\hline $\mathrm{T} 10$ & 150 & 50 & 50 \\
\hline $\mathrm{T} 11$ & 150 & 50 & 100 \\
\hline $\mathrm{T} 12$ & 150 & 50 & 150 \\
\hline $\mathrm{T} 13$ & 150 & 50 & 100 \\
\hline $\mathrm{T} 14$ & 150 & 50 & $100+\mathrm{Zn}$ \\
\hline $\mathrm{T} 15$ & 150 & 50 & $100+\mathrm{B}$ \\
\hline $\mathrm{T} 16$ & 150 & 50 & $100+\mathrm{Zn}+\mathrm{B}$ \\
\hline
\end{tabular}


Diseño Bloques Completos al Azar con 16 tratamientos $\mathrm{y}$ tres repeticiones.

Los resultados se sometieron al análisis de la varianza y las medias de los tratamientos comparadas con la prueba de Duncan al $5 \%$ de probabilidad de error estadístico.

Cada parcela estuvo conformada por 16 plantas, de las cuales, se evaluó 4 del área útil. Marco de plantación de 3 x 5 entre líneas y plantas respectivamente.

\subsection{Manejo del experimento}

Desbroce y limpieza de terreno. Semillero en fundas plásticas de 9” x 12”, depositando una semilla, en sustrato preparado con tierra y materia orgánica descompuesta. Hoyado siguiendo la distancia de siembra, 3 x $5 \mathrm{~m}$. Trasplante, 12 semanas después de la siembra. Construcción de espalderas utilizando estacas de muyuyo. Fertilización de acuerdo con los tratamientos. Control de plagas y enfermedades con productos amigables con el ambiente. Riego una vez por semana. Control de malezas, manual. Cosecha manual de los frutos maduros (amarillos).

Variables evaluadas: número de frutos por planta, peso por fruto, rendimiento por hectárea

\section{Resultados y discusión}

El análisis de la varianza en todas las variables de producción muestra dos grupos estadísticos., cuadro 3 El número de frutos por planta fluctúa entre 101,53 y 178,28; peso del fruto entre 124,73 y 162,2 gramos; todos los tratamientos donde se aplicó NPK y NPK mas microelementos muestran comportamiento similar en cuanto al rendimiento; la media general se sitúa en 13,5 t/ha/año, sobresaliendo el T9 con 15,5 t/ha/año, cuadro 3. Los resultados concuerdan con las investigaciones obtenidas por Joao et al (7) ; Aular J. y Rojas E. (8) e INIAP (9) quienes reportan peso del fruto, 91-102 gramos y rendimiento anual, 13,7 - 17,7 t/ha/año.

Los coeficientes de variación denotan la confiabilidad del experimento.

\subsection{Análisis económico}

El análisis marginal descarta los tratamientos dominados (Cuadro 4), siendo el tratamiento 6 el que muestra la mayor tasa de retorno marginal con $127941,53 \%$ (se considera $100 \%$, como Tasa de Retorno Mínima Aceptable), seguido del tratamiento 11 con 4069,56 \%.

\begin{tabular}{|l|lll|lll|ll|}
\hline \multicolumn{1}{|l|}{ Cuadro 3. Variables agronómicas. Test de Duncan } \\
\hline Trat. & $\begin{array}{l}\text { Frutos } \\
\text { planta. }\end{array}$ & por & Peso & del & \multicolumn{2}{l}{$\begin{array}{l}\text { Rendimiento } \\
\text { frutha }\end{array}$} & \\
\hline T1 & 101,53 & a & 124,73 & a & 8644,47 & a \\
\hline T2 & 126,7 & ab & 162,1 & b & 12190,57 & ab \\
\hline T3 & 158,37 & b & 150,33 & ab & 14601,1 & b \\
\hline T4 & 143,83 & ab & 147,27 & ab & 13182,77 & ab \\
\hline T5 & 126,37 & ab & 133,53 & ab & 11054,97 & ab \\
\hline T6 & 149,83 & ab & 149,53 & ab & 14106,7 & b \\
\hline T7 & 132,87 & ab & 155,87 & ab & 13760 & ab \\
\hline T8 & 151,27 & ab & 162,2 & b & 14680 & b \\
\hline T9 & 172,87 & b & 142,9 & ab & 15574,47 & b \\
\hline T10 & 161,33 & b & 140,1 & ab & 14834,47 & b \\
\hline T11 & 144,53 & ab & 145,77 & ab & 13322,8 & ab \\
\hline T12 & 178,27 & b & 134,23 & ab & 15380,57 & b \\
\hline T13 & 163 & b & 150,87 & ab & 15098,9 & b \\
\hline T14 & 143,53 & ab & 142,23 & ab & 13244,47 & ab \\
\hline T15 & 148,17 & ab & 136,53 & ab & 12965 & ab \\
\hline T16 & 156,7 & ab & 147,7 & ab & 14611,1 & b \\
\hline CV & 19,55 & & 11,4 & & 20,32 & \\
\hline
\end{tabular}

Cuadro 4. Análisis marginal del experimento

\begin{tabular}{|c|c|c|c|c|c|c|}
\hline Trat & $\begin{array}{c}\text { Costos } \\
\text { que varían }\end{array}$ & $\begin{array}{c}\text { Costos } \\
\text { Marginal }\end{array}$ & $\begin{array}{c}\text { Beneficio } \\
\text { Neto }\end{array}$ & $\begin{array}{c}\text { Beneficio neto } \\
\text { marginal }\end{array}$ & $\begin{array}{c}\text { Tasa de } \\
\text { retorno } \\
\text { marginal }\end{array}$ & $\begin{array}{c}\text { Tasa de } \\
\text { retorno } \\
\text { mínima } \\
\text { aceptable }\end{array}$ \\
\hline T1 & 0 & & 2586,86 & & & 100 \\
\hline & & 926,00 & & 135,17 & 14,59 & \\
\hline T2 & 926,00 & & 2722,03 & & & 100 \\
\hline & & 8,13 & & 330,69 & 4069,56 & \\
\hline T11 & 934,12 & & 3052,73 & & & 100 \\
\hline & & 0,18 & & 234,40 & 127941,53 & \\
\hline T6 & 934,31 & & 3287,12 & & & 100 \\
\hline & & 12,21 & & 205,58 & 1683,64 & \\
\hline T10 & 946,52 & & 3492,70 & & & 100 \\
\hline & & 54,11 & & 25,02 & 46,22 & \\
\hline T13 & 1000,62 & & 3517,72 & & & 100 \\
\hline & & 66,50 & & 17,79 & 26,75 & \\
\hline T12 & 1067,12 & & 3535,51 & & & 100 \\
\hline
\end{tabular}

\section{Conclusiones y recomendaciones}

\section{Conclusiones}

Del análisis estadístico de las variables agronómicas evaluadas se concluye:

- La Prueba de Rangos Múltiples de Duncan, muestra dos grupos para las variables peso del fruto, frutos por planta y rendimiento.

- Las dosis de fertilizantes influyen positivamente en el comportamiento productivo del cultivo.

- Los tratamientos 6, 10 y 11 superan la tasa de retorno mínima aceptable considerada en $100 \%$. 


\section{Recomendaciones}

- Para la zona de San Vicente de Colonche, se recomienda los tratamientos $\mathrm{T} 6\left(\mathrm{~N}_{150} ; \mathrm{P}{ }_{40} ; \mathrm{K}_{100}\right)$ y T11 $\left(\mathrm{N}_{150} ; \mathrm{P}_{50} ; \mathrm{K}_{50}\right)$.

- Validar la presente investigación en otros ambientes de la provincia de Santa Elena, con los tratamientos 6 y 11 considerando diferentes láminas de riego.

\section{Bibliografía}

1. III Censo Agropecuario. 2000. MAGAP .En línea. Consultado el 20 de feb. 2010. Disponible en http://www.sica.gov.ec/agronegocios/productos\%20par a\%20invertir/frutas/maracuya/presentacion_maracuy\% C3\%A1.htm

2. Ecuaquímica. 2010. El cultivo de maracuyá. En línea. Consultado el 18 de feb. 2010. Disponible en http://www.ecuaquimica.com/index.php?option=com_c ontent\&task=view\&id=26\&Itemid=28\&tit=Maracuy\% E1\&lang=

3. Diario Hoy. 2009. Maracuyá pierde su fuerza exportadora. Publicado el 16 de noviembre de 2009. Consultado el 5 de febrero de 2010. Disponible en: http://www.hoy.com.ec/noticias-ecuador/maracuyapierde-su-fuerza-exportadora-382570.html

4. Elizalde MF. 2011. Maracuyá Postcosecha. Universidad técnica de Machala. Facultad de Ciencias Agropecuaria, carrera de economía agropecuaria. Consultado el marzo de 2011. Disponible en: http://es.scribd.com/doc/49393358/poscosechamaracuya-FINAL

5. Malavolta E. 1994. Nutrición y fertilización del maracuyá. En línea. Consultado el 15 de feb. 2010. Disponible

en http://www.ipni.net/ppiweb/ltamn.nsf/87cb8a98bf7257 2b8525693e0053ea70/eb7e576f71ac1c3f05257068006 25d6b/\$FILE/NUTRICI\%C3\%93N\%20Y\%20FERTIL IZACI\%C3\%93N\%20DEL\%20MARACUYA.pdf

6. León Mejía A. 1996. Respuesta del Maíz (Zea maíz) hibrido Pacific 9205 a tres niveles de fertilización con fósforo y potasio en la península de Santa Elena. Tesis de Ing. Agr. Universidad Agraria Del Ecuador.

7. Joao LC. et al. 1991. Efecto de tres dosis de fertilización de NPK en la producción de maracuyá amarillo. Consultado el 20 de feb. 2010. Disponible en http://webnotes.sct.embrapa.br/pdf/pab1991/m arco/pab03_mar_91.pdf

8. Aular JY y Rojas E. 1992. Influencia del nitrógeno sobre el crecimiento vegetativo y producción de la parchita Passiflora edulis

Sims F. Flavicarpa Degener. en línea. Consultado el 19 de feb. 2010. Disponible en http://sian.inia.gob.ve/repositorio/revistas_ci/Agronomi a\%20Tropical/at4401/Arti/aular_j.htm

9. INIAP 2009. Manejo del cultivo de maracuyá en el litoral. Proyecto mejoramiento de la productividad y calidad de la fruticultura de la región sierra, litoral y
Amazonía. Programa de Fruticultura Estación experimental Portoviejo. Boletín divulgativo Nº 365.

\section{Bibliografía no referenciada.}

1. EL CULTiVO DE LA MARACUYÁ. sf. Fertilización del cultivo de maracuyá. En línea. Consultado el 17 de feb. 2010. Disponible en .http://www.encolombia.com/economia/Cultivos/Fertili zacionydeschuponadodemaracuya.htm

2. Ministerio de agricultura, Ganadería y Pesca Magap. 2010. Dirección provincial de santa Elena.

3. Molina E.; Salas SR. y Villalobos R. 1996. Efecto del magnesio en el rendimiento del Maracuyá. Nota técnica. En línea. Consultado el 20 de feb. 2010. Disponible

en

http://www.mag.go.cr/rev_agr/v20n01_053.pdf. 